\title{
Single-parameter pumping in graphene
}

\author{
Pablo San-Jose, ${ }^{1}$ Elsa Prada, ${ }^{2}$ Sigmund Kohler, ${ }^{2}$ and Henning Schomerus ${ }^{3}$ \\ ${ }^{1}$ Instituto de Estructura de la Materia (IEM-CSIC), Serrano 123, E-28006 Madrid, Spain \\ ${ }^{2}$ Instituto de Ciencia de Materiales de Madrid (ICMM-CSIC), Cantoblanco, E-28049 Madrid, Spain \\ ${ }^{3}$ Department of Physics, Lancaster University, Lancaster, LA1 4YB, United Kingdom
}

(Received 19 September 2011; published 11 October 2011)

\begin{abstract}
We propose a quantum pump mechanism based on the particular properties of graphene, namely chirality and bipolarity. The underlying physics is the excitation of evanescent modes entering a potential barrier from one lead, while those from the other lead do not reach the driving region. This induces a large nonequilibrium current with electrons stemming from a broad range of energies, in contrast to the narrow resonances that govern the corresponding effect in semiconductor heterostructures. Moreover, the pump mechanism in graphene turns out to be robust, with a simple parameter dependence, which is beneficial for applications. Numerical results from a Floquet scattering formalism are complemented with analytical solutions for small to moderate driving.
\end{abstract}

DOI: 10.1103/PhysRevB.84.155408

PACS number(s): 72.80.Vp, 05.60.Gg, 72.40.+w

\section{INTRODUCTION}

Ratchets and pumps are devices in which spatiotemporal symmetry breaking turns an ac force without net bias into directed motion. ${ }^{1,2}$ If the time-dependence enters via only one parameter, the pump current vanishes in the adiabatic limit. ${ }^{3}$ Therefore, single-parameter pumping requires nonequilibrium conditions enforced by driving beyond the adiabatic limit. This distinguishes single-parameter pumps from devices that operate with oriented work cycles, like turnstiles ${ }^{4}$ or sluices. $^{5}$ Quantum pumps can be implemented with quantum dots in a two-dimensional electron gas (2DEG) driven by microwaves, ${ }^{6}$ surface-acoustic waves, ${ }^{7}$ ac gate voltages, ${ }^{8-11}$ or nonequilibrium noise. ${ }^{12}$ Here we propose a single-parameter pump based on the particular properties of graphene and show that these display a broad-band response, in contrast to 2DEG-based ratchets or pumps for which isolated resonances govern the effect. ${ }^{6,12,13}$

Owing to the chiral and gapless nature of charge carriers in graphene, an electron hitting a potential step in this material may propagate forward as a hole with opposite momentum. ${ }^{14,15}$ Due to this Klein tunneling, it is difficult to confine electrons by electrostatic potentials. This phenomenon even extends to evanescent modes, i.e., modes that decay exponentially as a function of the barrier penetration. In graphene, electrons populating such modes can tunnel a large distance. ${ }^{16}$ This impediment to electrostatic confinement constitutes a drawback for many switching and sensing applications.

An inspection of present quantum pump designs suggests that graphene pumps would be negatively affected by this issue as well. For example, pumps have been realized with electrostatically defined double quantum dots in a 2 DEG. ${ }^{6,12}$ In these experiments, a driving field induces dipole transitions of an electron from a metastable state below the Fermi energy in the, say, left dot to a metastable state in the right dot. Subsequently, the electron will leave to the right lead, and an electron from the left lead will fill the empty state in the left dot. The emerging pump current therefore requires resonant conditions with a pair of energetically well-defined states, i.e., well isolated states with long life times, which do not exist in graphene.

Nevertheless, the alternative, graphene-specific mechanism identified here allows realizing highly efficient pumping, which may indeed outperform conventional devices. The large pump current emerges in the bipolar regime around the Dirac point. This is due to a scattering process where a whole continuum of evanescent modes is promoted into unidirectionally propagating states, which couple well to the leads because of chirality. Since this mechanism does not rely on intricate resonance conditions, but invokes graphene's intrinsic features of bipolarity and chirality, the pump current is robust and displays a simple parameter dependence curve, as is desirable for electronic sensor applications.

This paper is organized as follows: In the next section we describe our proposal for a single-parameter pump and give typical values for length and energies scales. In Sec. III we introduce the Floquet scattering theory that applies to nonequilibrium pumping in graphene. We first present the general formalism and then consider the weak driving regime, where we derive analytical expressions for the one-photon transmission probability. In Sec. IV we present numerical results for the pumped current comparing the performances of graphene and a 2DEG pump. Moreover, we compare the numerics with the semiclassical approximation derived in the preceding section and find good agreement. The specific pumping mechanism by excitation of evanescent modes is explained in Sec. V. Finally, we conclude in Sec. VI.

\section{DESCRIPTION OF THE SYSTEM}

As a single-parameter pump setup, sketched in Fig. 1(a), we consider a graphene ribbon of length $2 L$ and width $W$ attached to two metallic electron reservoirs, with its left half driven by a time-dependent gate. The driving by variation of only one parameter excludes the emergence of an adiabatic pump current. ${ }^{3,17}$ However, considering that spatial symmetry is broken by the placement of the gate, a finite dc current arises in nonequilibrium conditions, which can be achieved by nonadiabatic driving. In order to assess the importance of graphene's chirality and bipolarity in the nonadiabatic context, we contrast our results with those found for the corresponding setup of a 2DEG in a semiconductor heterostructure.

We assume that the sample sizes are smaller than the mean free path and that $W \gg L$, so that the effects coming from the boundaries, electron-electron interactions, and disorder play 

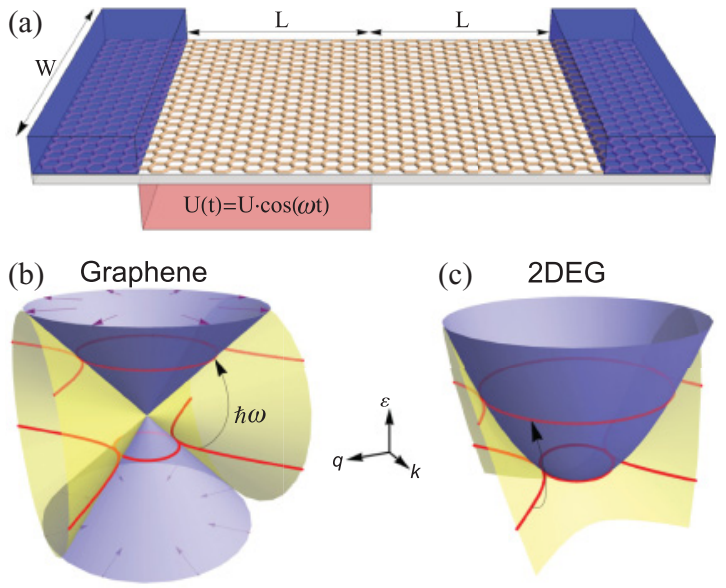

(c) 2DEG

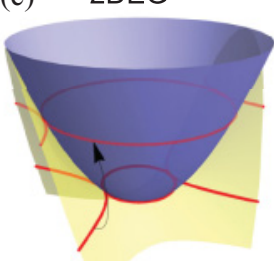

FIG. 1. (Color online) Sketch of a single-parameter graphene pump connected to two leads. The left half of the graphene ribbon is exposed to an ac gate voltage. Dispersion of (b) graphene and (c) a 2DEG, with longitudinal and transverse momenta $k$ and $q$, including the branches of evanescent modes. Photon absorption (and in the case of graphene also emission) may excite an evanescent mode to propagating.

a minor role. Besides, we restrict ourselves to the low-energy regime such that the Dirac approximation remains valid. Then, our quasi one-dimensional model contains three natural energy scales, namely the driving frequency $\omega$, the driving amplitude $U$, and the energy associated with the length $L$ of the device. Additionally, the leads' Fermi momentum $\hbar k_{F}^{(\infty)}$ becomes a relevant scale in a $2 \mathrm{DEG}$, since the contact resistance depends on its value. For graphene with Fermi velocity $v_{F} \approx 10^{6} \mathrm{~m} / \mathrm{s}$ and $L=5 \mu \mathrm{m}$, the latter is $E_{L}^{G}=\hbar v_{F} / L \approx$ $0.13 \mathrm{meV}$. For a $2 \mathrm{DEG}$ with the same geometry and effective mass $m^{*}=0.067 m_{\mathrm{e}}(\mathrm{GaAs} / \mathrm{AlGaAs})$, it is roughly four orders of magnitude smaller, $E_{L}^{N}=\hbar^{2} / 2 m^{*} L^{2} \approx 0.02 \mu \mathrm{eV}$. Driving beyond the adiabatic limit requires frequencies $\omega \gtrsim E_{L} / \hbar$. Electrons can then absorb or emit photons, and after a short transient period, these excitations will establish a nonequilibrium population of the electronic states.

\section{FLOQUET SCATTERING THEORY IN GRAPHENE}

A quantitative description of nonadiabatic charge transport is provided by Floquet scattering theory. Subsequently, we show that this theory provides the probability $T_{L R}^{(n)}$ for an electron to be scattered from the left to the right lead under the absorption or emission of $n$ photons, and with it we can calculate the dc current. ${ }^{18}$ In order to focus on the graphene-specific features, we will restrict ourselves to the zero temperature limit so that all electronic states below the Fermi energy $E_{F}$ are initially occupied.

\section{A. General formalism}

When scattered at a periodically time-dependent potential, an electron with initial energy $\epsilon$ may absorb or emit $|n|$ quanta of the driving field ( $n<0$ corresponds to emission), such that its final energy is $\epsilon+n \hbar \omega$. This is embodied in the decomposition of the transmission probability from the left to the right reservoir:

$$
T_{L R}(\epsilon)=\sum_{n=-\infty}^{\infty} T_{L R}^{(n)}(\epsilon)
$$

The corresponding time-averaged dc current is given by the generalized Landauer formula, ${ }^{18,19}$

$$
\bar{I}=\frac{g e}{h} \int d \epsilon \sum_{n}\left[T_{L R}^{(n)}(\epsilon) f_{L}(\epsilon)-T_{R L}^{(n)}(\epsilon) f_{R}(\epsilon)\right],
$$

where $e$ is the electron charge and $f(\epsilon)$ is the Fermi-Dirac distribution. Spin and valley degeneracy of graphene is responsible for the prefactor $g=4$, while $g=2$ accounts for the spin in a 2DEG. The application of Eq. (2) to graphene (or any other two-dimensional material) requires extending the summation to transverse momenta $\hbar q$. For a driving field that breaks reflection symmetry, one generally finds $T_{R L}(\epsilon) \neq$ $T_{L R}(\epsilon)$. Then, even when the leads are in equilibrium, such that $f_{L}(\epsilon)=f_{R}(\epsilon) \equiv f(\epsilon)$, a net current may flow, and a pump current emerges,

$$
\bar{I}=\frac{g e}{h} \int d \epsilon f(\epsilon) \Delta T(\epsilon),
$$

where $\Delta T=T_{L R}-T_{R L}$.

For the computation of the transmission probabilities we adopt the Floquet scattering formalism of Ref. 20 and consider electrons in two dimensions under the influence of a timedependent potential described by the Hamiltonian

$$
H(t)=H_{0}(x)+U(x) \cos (\omega t) .
$$

Here, $H_{0}$ comprises the kinetic energy and the static potential $V(x)$, while $U(x)$ is the profile of the time-dependent potential with frequency $\omega$. Since the potential is $y$-independent, the transverse momentum is conserved and the problem becomes effectively one-dimensional. We assume that both the static potential $V(x)$ and the driving profile $U(x)$ are piecewise constant, and that $U(x)=0$ outside the scattering region; see Fig. 2. For graphene, $H_{0}=\hbar v_{F} \boldsymbol{k} \cdot \boldsymbol{\sigma}+V(x)$ with the

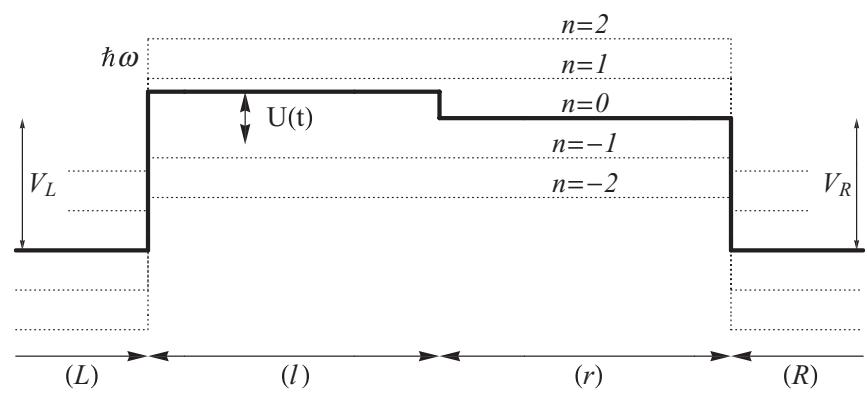

FIG. 2. Quasi-one-dimensional scattering potential. Profile of the pump, modeled as a barrier for which the left half (region $l$ ) experiences an oscillatory gate voltage. Regions $L$ and $R$ correspond to highly doped leads. Dotted lines denote sidebands to which the electron energy changes by absorption and emission of photons from the driving field. 
wavevector $\boldsymbol{k}= \pm k \boldsymbol{e}_{x}+q \boldsymbol{e}_{y}$. The free solution with energy $E$ reads

$$
\varphi_{E}^{ \pm}=\frac{e^{ \pm i k x}}{\sqrt{2|E| k / \hbar}}\left(\begin{array}{c}
|E| / \hbar v_{F} \\
\pm k+i q
\end{array}\right),
$$

where $k$ is positive and fulfills the dispersion relation $E^{2}=$ $\hbar^{2} v_{F}^{2}\left(k^{2}+q^{2}\right)$, while \pm is the sign of the corresponding current. The normalization has been chosen such that propagating waves have unit longitudinal current, $v_{F}\left(\varphi_{E}^{ \pm}\right)^{\dagger} \sigma_{x} \varphi_{E}^{ \pm}= \pm 1$. This is convenient since then the coefficients of a superposition become probability amplitudes. For evanescent solutions with imaginary longitudinal wavenumber $k=i \kappa$, the current vanishes.

According to the Floquet theorem, the Schrödinger equation with a time-periodic Hamiltonian $H(t)=H(t+2 \pi / \omega)$ possesses a complete set of solutions with structure $\psi=$ $e^{-i \epsilon t / \hbar} \phi(t)$, where the Floquet state $\phi(t)=\phi(t+2 \pi / \omega)$ obeys the time-periodicity of the Hamiltonian and $\epsilon$ is the quasienergy. Here we are looking for Floquet scattering states, i.e., solutions of the Schrödinger equation that (i) are of Floquet structure and (ii) have an incoming plane wave as boundary condition. For clarity, we derive here only the transmission from left to right-for the opposite direction, it follows by simple relabeling.

Condition (i) is equivalent to employing for the wavefunction in any of the four regions $\ell=L, l, r, R$, the ansatz $\psi_{\ell}(x, t)=e^{-i \epsilon t / \hbar} \phi_{\ell}(x, t)$. The time-periodic parts $\phi_{\ell}(x, t)$ still have to be determined, while the quasienergy $\epsilon$ turns out to equal the energy of the incoming wave. Inserting $\psi_{\ell}$ into the Schrödinger equation of region $\ell$ yields for $\phi_{\ell}(x, t)$ a partial differential equation, which we solve by a separation ansatz. The resulting solutions

$$
\begin{aligned}
\phi_{n, \ell}^{ \pm}(x, t) & =e^{-i n \omega t-i\left(U_{\ell} / \hbar \omega\right) \sin (\omega t)} \varphi_{\epsilon+n \hbar \omega-V_{\ell}}^{ \pm}(x) \\
& =\sum_{n^{\prime}=-\infty}^{\infty} J_{n^{\prime}-n}\left(U_{\ell} / \hbar \omega\right) e^{-i n^{\prime} \omega t} \varphi_{\epsilon+n \hbar \omega-V_{\ell}}^{ \pm}(x),
\end{aligned}
$$

comply with the requirement of time-periodicity provided that the separation parameter $n$ is of integer value. The separation parameter labels all possible solutions and determines the time-averaged energy $\epsilon+n \hbar \omega$. The Bessel function of the first kind $J_{n}$ stems from the relation $\exp [-i z \sin (\omega t)]=$ $\sum_{n} J_{n}(z) \exp (-i n \omega t)$. Note that in the present case, $U_{\ell}$ is nonzero only in the driving region $\ell=l$. The ansatz Eq. (6) has also been used to study photo-assisted tunneling in graphene. ${ }^{21,22}$

Condition (ii) means that in region $L$, the Floquet state consists of an incoming plane wave and a reflected part, $\phi_{L}=\varphi_{\epsilon-V_{L}}^{+}+\sum_{n} r_{n} \phi_{n, L}^{-}$, while in region $R$, we have only an outgoing state, $\phi_{R}=\sum_{n} t_{n} \phi_{n, R}^{+}$. With the normalization chosen for the free solutions, Eq. (5), the coefficients of the latter superposition relate to the left-to-right transmission probability under absorption or emission of $n$ quanta according to

$$
T_{L R}^{(n)}(\epsilon)=\left|t_{n}(\epsilon)\right|^{2} .
$$

In the scattering regions $l$ and $r$, the Floquet solution must be a superposition of the states of Eq. (6); i.e., $\phi_{\ell}=\sum_{n} t_{n}^{(\ell)} \phi_{n, \ell}^{+}+\sum_{n} r_{n}^{(\ell)} \phi_{n, \ell}^{-}$. The coefficients $t_{n}^{(\ell)}$ and $r_{n}^{(\ell)}$ follow from the requirement that the scattering states have to be continuous at any time. These matching conditions can be written as a set of linear equations, with the incoming wave appearing as inhomogenity. In matrix notation it reads

$$
\sum_{n^{\prime}} M_{n, n^{\prime}} \cdot\left(r_{n^{\prime}}, t_{n^{\prime}}^{(l)}, r_{n^{\prime}}^{(l)}, t_{n^{\prime}}^{(r)}, r_{n^{\prime}}^{(r)}, t_{n^{\prime}}\right)=\delta_{n, 0} \tilde{\varphi}_{L, \text { in }}^{+},
$$

where $\tilde{\varphi}_{L, \text { in }}^{+} \equiv \varphi_{\epsilon-V_{L}}^{+}$denotes the incoming wave written as a 6 -dimensional vector. The $6 \times 6$ matrices $M_{n, n^{\prime}}$ are constructed from the wavefunctions of Eq. (6) evaluated at the interfaces. They can be written efficiently as

$$
M_{n, n^{\prime}}=M\left(\epsilon-n^{\prime} \hbar \omega\right) \sum_{\ell} J_{n^{\prime}-n}\left(U_{\ell} / \hbar \omega\right) P_{\ell},
$$

where $M(\epsilon)$ contains the wave-matching condition at energy $\epsilon$ in the absence of driving. The matrix $P_{\ell}$ is a projector to region $\ell$, constructed such that $M P_{\ell}$ contains only wavefunctions from region $\ell$. In particular, $P_{l}=\operatorname{diag}(0,1,1,0,0,0)$ projects onto the driving region. Equation (8) fully determines the transmission and reflection amplitudes $t_{n}$ and $r_{n}$. The tight-binding version ${ }^{18}$ of this method is suitable for studying ac driving of smaller carbon-based conductors, such as ribbons with a size of only a few lattice constants ${ }^{23}$ or nanotubes. ${ }^{24,25}$ Via time-dependent density functional theory, it can be generalized to the presence of interactions. ${ }^{26}$

For electrons in a 2DEG with effective mass $m^{*}$, the static Hamiltonian reads $H_{0}=\hbar^{2} \boldsymbol{k}^{2} / 2 m^{*}+V(x)$. Its free solutions are scalar fields, which must be continuous. But since their derivatives must also be continuous, it is convenient to write them in spinor notation

$$
\varphi_{E}^{ \pm}=\frac{e^{ \pm i k x}}{\sqrt{\hbar k / m^{*}}}\left(\begin{array}{c}
1 \\
\pm i k
\end{array}\right),
$$

with the dispersion relation $E=\hbar^{2} k^{2} / 2 m^{*}$. The normalization is such that the first vector component has unit current, $\frac{1}{m^{*}}\left(\varphi_{E, 1}^{ \pm}\right)^{\dagger}\left(-i \hbar \partial_{x}\right) \varphi_{E, 1}^{ \pm}= \pm 1$. With these ingredients, the Floquet-scattering formalism can be directly applied to the 2DEG case.

\section{B. Weak driving limit}

The set of linear Eqs. (8) can be solved analytically in the limit of small driving amplitudes or large frequencies, such that $p \equiv(U / 2 \hbar \omega)^{2} \ll 1$. We aim at finding analytical expressions for the one-photon transmission probabilities $T^{( \pm 1)}$ in terms of the static transmission for Klein tunneling at energy $\epsilon$ with respect to the top of a high barrier ${ }^{15}$ of length $L$,

$$
T(\epsilon, q)=\frac{k_{\epsilon}^{2}}{k_{\epsilon}^{2}+q^{2} \sin ^{2}\left(k_{\epsilon} L\right)},
$$

where $k_{\epsilon}=\left[\left(\epsilon / \hbar v_{F}\right)^{2}-q^{2}\right]^{1 / 2}$. The semiclassical limit $L \rightarrow$ $\infty$ of the above relation is obtained by integration over fast oscillations, which yields $T(\epsilon, q) \approx \sqrt{1-\left(\hbar v_{F} q / \epsilon\right)^{2}}$. In order to relate these expressions to our formalism, we extract from the solution of Eq. (8) in the undriven limit, $U=0$, the transmission amplitude $t_{0}$ by multiplication with the vector $\mathrm{p}_{R}^{\dagger}=(0,0,0,0,0,1)$ and obtain

$$
T(\epsilon)=\left|\mathrm{p}_{R}^{\dagger} M^{-1}(\epsilon) \tilde{\varphi}_{L, \text { in }}^{+}\right|^{2} .
$$


Next, we simplify Eq. (8) using for the Bessel functions the approximations $J_{0}(U / \hbar \omega)=1, J_{ \pm 1}(U / \hbar \omega)= \pm U / 2 \hbar \omega$, while $J_{n}(U / \hbar \omega)=\mathcal{O}^{|n|}(U / \hbar \omega)$ for $U / \hbar \omega \ll 1$. Thus, to first order in $U / \hbar \omega$, only the Floquet indices $n=0, \pm 1$ remain. The resulting $18 \times 18$ matrix $\left(M_{n, n^{\prime}}\right)$ can be inverted via the approximation $[A+(U / 2 \hbar \omega) B]^{-1}=A^{-1}-$ $(U / 2 \hbar \omega) A^{-1} B A^{-1}$, which provides $t_{ \pm 1}$ and, thus,

$$
T_{L R}^{( \pm 1)}=p\left|\mathrm{p}_{R}^{\dagger} M^{-1}(\epsilon \mp \hbar \omega) M(\epsilon) P_{l} M^{-1}(\epsilon) \tilde{\varphi}_{L, \text { in }}^{+}\right|^{2} .
$$

The calculation of the elastic transmission $T_{L R}^{(0)}$ up to leading order in $p$ is more tedious, but fortunately not required, because our Hamiltonian obeys time-reversal symmetry. Therefore, $T_{L R}^{(0)}(\epsilon)=T_{R L}^{(0)}(\epsilon)$, which implies that the elastic channel does not contribute to the pump current. ${ }^{18}$

Our numerical calculations will reveal that the relevant contributions to the pump current stem from modes that, in the absence of driving, are evanescent in the barrier region. For such modes, Eq. (13) can be connected to Eq. (12) in a simple way, because the modified source term in Eq. (13) has an invariant forward amplitude, $M(\epsilon) P_{l} M^{-1}(\epsilon) \tilde{\varphi}_{L, \text { in }}^{+}=$ $\tilde{\varphi}_{L, \text { in }}^{+}+\alpha^{-} \tilde{\varphi}_{L, \text { in }}^{-}$. Although $\alpha^{-}$is not necessarily zero, it does not affect the transmission, since it merely redefines the reflection amplitude $r_{0}$. As a result, the one-photon transmission, Eq. (13), becomes, besides a prefactor $p$, identical to the static transmission of Eq. (12) in the evanescent region:

$$
T_{L R}^{( \pm 1)}(\epsilon)=p T(\epsilon \pm \hbar \omega, q) .
$$

For the evanescent modes entering from the right, the same reasoning lets us conclude that the waves decay exponentially and do not reach the driven region $l$. Consequently, $P_{l} M^{(-1)}(\epsilon) \tilde{\varphi}_{R \text {,in }}^{-} \approx 0$ and $T_{R L}^{( \pm 1)}(\epsilon) \ll p$ can be neglected. Then the net transmission appearing in Eq. (3) becomes

$$
\Delta T=p[T(\epsilon+\hbar \omega, q)+T(\epsilon-\hbar \omega, q)]+\mathcal{O}\left(e^{-2 \kappa L}\right) .
$$

The magnitude of the correction reflects the fact that we have neglected the exponentially small transmission of the evanescent modes, which decays with the imaginary wavenumber $i \kappa=i\left[q^{2}-\left(\epsilon / \hbar v_{F}\right)^{2}\right]^{1 / 2}$. Since $0 \leqslant T \leqslant 1$, it follows that the maximal net transmission for evanescent waves is $2 p$.

\section{NONADIABATIC PUMP CURRENT}

In the relevant weak driving regime $U \ll \hbar \omega$, the absorption or emission probabilities are of the order $p \equiv(U / 2 \hbar \omega)^{2} \ll 1$ [see Eq. (14)], so that $\Delta T \sim p$. Moreover, for short and wide systems (width $W \gg L$ ), the total current takes the form of an integral over modes $q$. Hence, the current Eq. (3) at a given Fermi energy $E_{F}$ (measured from the Dirac point of the barrier) can be expressed as

$$
\bar{I}=\frac{g e}{h}\left(\frac{U}{2 \hbar \omega}\right)^{2} W \int_{-\infty}^{E_{F}} d \epsilon \int_{-\infty}^{\infty} d q \frac{\Delta T}{p} .
$$

We compute $\Delta T$ numerically by wave-matching in Floquet space [see Eq. (8)]. Using typical parameters $L=5 \mu \mathrm{m}$, $W / L=4, U=40 \mu \mathrm{eV}, \hbar \omega=2 \mathrm{meV}$ (around $500 \mathrm{GHz}$ ), we obtain the results shown in Fig. 3. As long as $p \ll 1$, the structure of the transmission for graphene depends only on the product $L \omega$, unlike for the 2DEG. Graphene develops a far

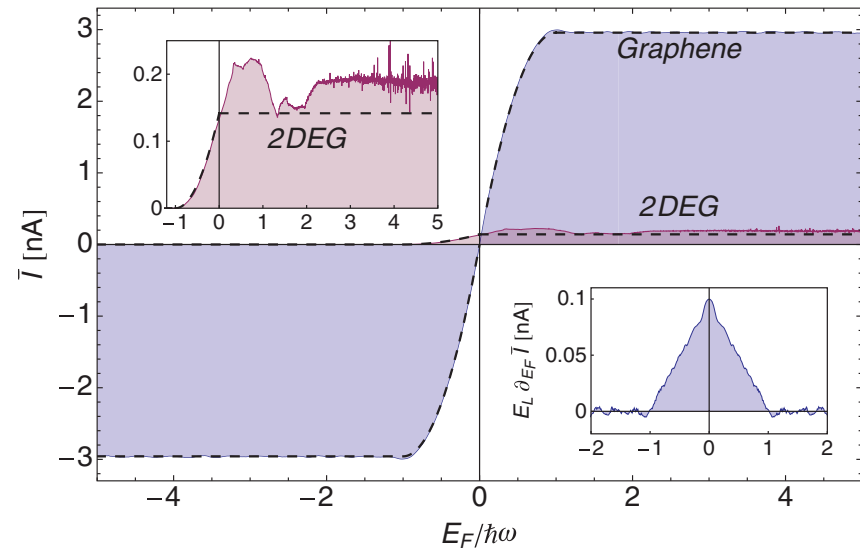

FIG. 3. (Color online) Pump current $\bar{I}$ for graphene and 2DEG. Dashed lines mark the semiclassical result, which closely matches the numerical ones. The upper inset is a blowup of the 2DEG case. The lower inset depicts the differential current, demonstrating that the main contribution to the pump current arises from an energy range $\hbar \omega$ around the Dirac point, populated by modes that are evanescent in the barrier region.

larger pump current than a $2 \mathrm{DEG}$, saturating to an $L$ - and $\omega$ independent maximal value $\bar{I}^{\max } \approx 3 \mathrm{nA}$ for $\left|E_{F}\right| \geqslant \hbar \omega$, while the smaller pump current in the 2DEG case already saturates at $\left|E_{F}\right| \gtrsim 0$. The semiclassical approximation described in Sec. III B, valid for $\hbar \omega \gg E_{L}$, yields

$$
\bar{I}_{G} \approx \frac{e}{\hbar} \frac{(U / 2)^{2}}{E_{W}^{G}} \times\left\{\begin{array}{ll}
\left(2-\frac{\left|E_{F}\right|}{\hbar \omega}\right) \frac{E_{F}}{\hbar \omega}, & \left|E_{F}\right|<\hbar \omega \\
\pm 1, & \left|E_{F}\right|>\hbar \omega
\end{array},\right.
$$

where the energy $E_{W}$ is the analog of $E_{L}$, with $L$ replaced by the width $W$. The corresponding calculation for a $2 \mathrm{DEG}$ provides the result

$$
\bar{I}_{N} \approx \frac{e}{\hbar} \frac{(U / 2)^{2}}{2 k_{F}^{(\infty)} W E_{W}^{N}} \times \begin{cases}0, & E_{F}<-\hbar \omega \\ \left(1+\frac{E_{F}}{\hbar \omega}\right)^{2}, & -\hbar \omega<E_{F}<0 . \\ 1, & E_{F}>0\end{cases}
$$

These approximations are plotted as dashed curves in Fig. 3. Unlike for graphene, the pump current in the 2DEG depends on the leads' large Fermi momentum $k_{F}^{(\infty)}$ (around $12 \mathrm{~nm}^{-1}$ for gold electrodes), which has been assumed equal for both leads. The relative pump performance, $v \equiv \bar{I}_{G}^{\max } / \bar{I}_{N}^{\max }=$ $\hbar k_{F}^{(\infty)} / m^{*} v_{F}$, assuming gold electrodes and GaAs/AlGaAs 2DEGs, is $v \approx 20$; i.e., the pump current in the graphene device is much larger than in the 2DEG device.

\section{DIRECTION-DEPENDENT EXCITATION OF EVANESCENT MODES}

In the following, we show that the large and robust pump current of the graphene device stems from a mechanism, whereby the ac field promotes evanescent modes from the left lead with probability $p$ into propagating modes, unlike evanescent modes from the right lead, which couple poorly to the driving region. In order to support this picture, we consider the differential response $d \bar{I} / d E_{F}$, shown in the lower inset of Fig. 3. It indicates that the main contribution to the current stems from the bipolar regime $|\epsilon|<\hbar \omega$ in the case of graphene, or from the gap boundary region $-\hbar \omega<\epsilon<0$ in 

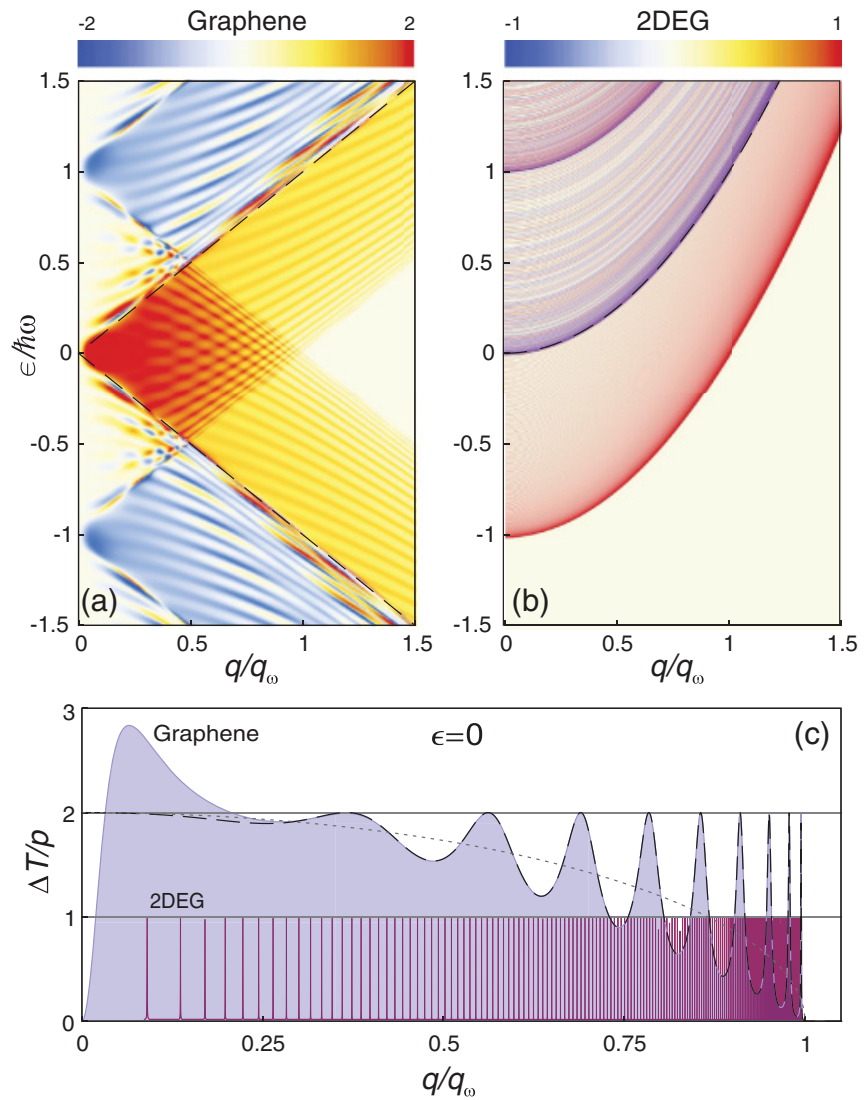

FIG. 4. (Color online) Scaled net transmission $\Delta T / p$, where $p=$ $(U / 2 \hbar \omega)^{2}$, for (a) the graphene and (b) the 2DEG pump as a function of transverse wave number $q$ and the initial energy $\epsilon$ for weak driving $U \ll \hbar \omega$ at photon energy $\hbar \omega=2 \mathrm{meV} \sim 500 \mathrm{GHz}$. The dashed lines separate regions with propagating and evanescent modes under the barrier. (c) Cut at zero energy revealing the behavior in the evanescent region. The dashed line marks the static transmission $2 T$ for graphene at energy $\hbar \omega$, while the dotted curve is its semiclassical approximation. The transverse wave number $q$ is scaled by $q_{\omega}$, which is defined by $q_{\omega} L=\hbar \omega / E_{L}^{G}$ for graphene, and $\left(q_{\omega} L\right)^{2}=\hbar \omega / E_{L}^{N}$ for the 2DEG.

the case of the 2DEG. In the absence of driving, these energy ranges are populated by electronic modes that become evanescent in the barrier region. Whether modes are evanescent or propagating furthermore depends on their transverse momentum $q$. Their response to driving is encoded in the function $\Delta T / p$, which represents the differential response for a given mode with transverse momentum $q$ and total energy $\epsilon$ [see Eq. (16)].

Figure 4 depicts $\Delta T / p$ for graphene and the 2DEG. In the $2 D E G$ case, at each energy only a discrete set of resonant modes contributes to the pump effect. These resonances correspond, up to a shift of $\pm \hbar \omega$ in energy, to quasibound levels in the static system, which form because of the velocity mismatch at the interface with the metallic leads, resulting in strong confinement. The contact resistance increases with increasing Fermi momentum $k_{F}^{(\infty)} L$, and the resonances become increasingly narrow, resulting in a suppressed response of the 2DEG. By contrast, in graphene a broad range of modes contributes at all energies, and the response is particularly strong in a diamond-shaped region

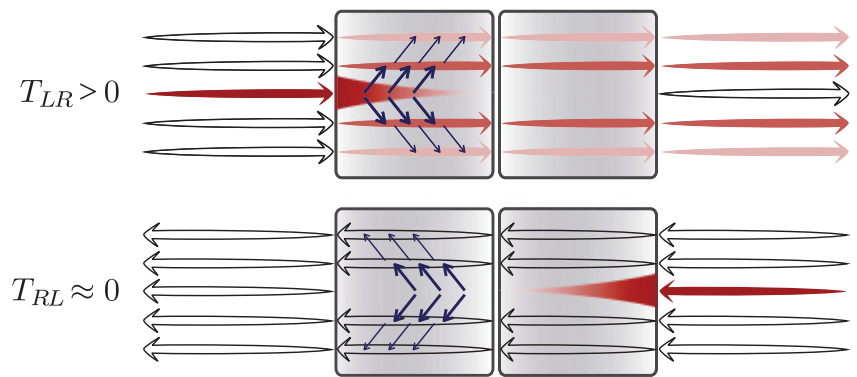

FIG. 5. (Color online) Evanescent mode pump mechanism. Evanescent modes penetrating the barrier from the left absorb or emit photons such that they become propagating. The corresponding modes from the right lead decay before reaching the region with the ac gating. Solid arrows indicate strong population; open arrows mark negligible scattering channels.

within the bipolar regime (red square in Fig. 4), in agreement with our earlier observation in Fig. 3. Graphene's response does not exhibit sharp resonances due to the fact that, in the static case, carrier chirality prohibits their confinement, so that carriers in the graphene pump remain strongly coupled to the leads, even when they are driven far out of equilibrium. Instead, graphene's response in Fig. 4(a) has features associated with the cone $\epsilon=\hbar v_{F} q$ (dashed lines) and its replicas shifted by multiples of $\hbar \omega$. For the weak driving considered here, only the two replicas at $\epsilon=\hbar v_{F} q \pm \hbar \omega$ are visible. The first cone divides the modes entering the scattering region into two categories, propagating and evanescent: any incoming carrier in mode $q$ will become evanescent under the barrier if its energy fulfills $|\epsilon|<\hbar v_{F} q$, and will remain propagating otherwise. The other two cones determine whether carriers populate evanescent or propagating modes after absorption or emission of a single photon. Analogously, in the 2DEG the threshold between propagating and evanescent modes is found at $\epsilon=\hbar^{2} q^{2} / 2 m^{*}$ and under photon emission or absorption shifts to $\epsilon=\hbar^{2} q^{2} / 2 m^{*} \pm \hbar \omega$.

In both systems, it can now be seen that the main mechanism of charge transfer is established by a process whereby an evanescent mode coming from the left lead may get transmitted to the right by absorbing or emitting a photon, as long as in this process it jumps to an existing propagating mode that may travel into the right lead. An evanescent coming from the right, in contrast, first encounters the static region not covered by the gate, and so gets reflected with a high probability (see Fig. 5). This spatial asymmetry rectifies the carrier flux such that a net transport from left to right emerges.

In the 2DEG, when photon absorption is at resonance with a quasibound state, this process via evanescent modes yields a maximal value $\Delta T / p \sim 1$, but because the resonances are narrow, the integrated current remains small. In graphene, on the other hand, all evanescent modes with $q L<\hbar \omega / E_{L}^{G}$ exhibit a response $\Delta T / p \lesssim 2$, close to twice the optimal value of resonant evanescent modes in a 2 DEG but without the need to satisfy any resonance condition. The factor two comes from the two possible transitions to a propagating mode, by absorption but also by emission of a photon; i.e., it is directly related to bipolarity. Resonant conditions are not required because Klein tunneling in the propagating modes and 
macroscopic tunneling in evanescent modes keep the contacts always open; this is directly related to chirality. In combination, these two graphene-specific effects maximize the differential pump response around the Dirac point and, thus, determine the robust characteristic features of the total pump current in Fig. 3.

\section{CONCLUSIONS}

With this work, we have put forward a pump mechanism that makes use of the evanescent modes under a potential barrier in graphene. If an ac gate voltage acts upon the, say, left half of the barrier, electrons penetrating the barrier from the left lead are excited into propagating modes and, thus, will be scattered to the right lead. By contrast, electrons from the opposite side will reach the driving region only with exponentially small probability. This breaking of spatiotemporal symmetries induces surprisingly large nonadiabatic pump currents in the range of several $\mathrm{nA}$, significantly larger than what has been observed so far with semiconductors. The main reason for this efficiency is that a whole range of evanescent modes is excited, while in the corresponding setup with a 2DEG, only isolated resonances contribute. Despite the large pump currents, the main effect stems from single-photon absorption, which allows one to obtain analytical results. Moreover, owing to current conservation, ratchets that are built by several pumps in series exhibit identical operation characteristics (repetition of the element is therefore not desirable for practical implementations).

A further important observation is that the resulting pump current changes smoothly, symmetrically, and almost monotonically with the distance of the Fermi energy to the Dirac point. This behavior is a direct consequence of bipolarity and, thus, particular to graphene pumps. As an application, it allows steering the dc current into a direction of choice by simply shifting the barrier height via a local dc gate voltage across the Dirac point. This effect can also be used to detect a static electric field by measuring the current in response to a small oscillating probe electric field, or detecting the amplitude of such an oscillating field when the static field is fixed to a moderately large value, beyond which the response flattens out.

\section{ACKNOWLEDGMENTS}

We acknowledge support by the CSIC JAE-Doc program and the Spanish Ministry of Science and Innovation through Grant Nos. FIS2008-00124/FIS (P.S.-J), FIS200908744 (E.P.), and MAT2008-02626 (S.K.).
${ }^{1}$ P. Reimann, Phys. Rep. 361, 57 (2002).

${ }^{2}$ P. Hänggi and F. Marchesoni, Rev. Mod. Phys. 81, 387 (2009).

${ }^{3}$ P. W. Brouwer, Phys. Rev. B 58, R10135 (1998).

${ }^{4}$ M. Switkes, C. M. Marcus, K. Campman, and A. C. Gossard, Science 283, 1905 (1999).

${ }^{5}$ J. J. Vartiainen, M. Mottonen, J. P. Pekola, and A. Kemppinen, Appl. Phys. Lett. 90, 082102 (2007).

${ }^{6}$ T. H. Oosterkamp, T. Fujisawa, W. G. van der Wiel, K. Ishibashi, R. V. Hijman, S. Tarucha, and L. P. Kouwenhoven, Nature (London) 395, 873 (1998).

${ }^{7}$ M. D. Blumenthal, B. Kaestner, L. Li, S. Giblin, T. J. B. M. Janssen, M. Pepper, D. Anderson, G. Jones, and D. A. Ritchie, Nature Phys. 3, 343 (2007).

${ }^{8}$ L. DiCarlo, C. M. Marcus, and J. S. Harris Jr., Phys. Rev. Lett. 91, 246804 (2003).

${ }^{9}$ B. Kaestner, V. Kashcheyevs, G. Hein, K. Pierz, U. Siegner, and H. W. Schumacher, Appl. Phys. Lett. 92, 192106 (2008).

${ }^{10}$ A. Fujiwara, K. Nishiguchi, and Y. Ono, Appl. Phys. Lett. 92, 042102 (2008).

${ }^{11}$ B. Kaestner, C. Leicht, V. Kashcheyevs, K. Pierz, U. Siegner, and H. W. Schumacher, Appl. Phys. Lett. 94, 012106 (2009).

${ }^{12}$ V. S. Khrapai, S. Ludwig, J. P. Kotthaus, H. P. Tranitz, and W. Wegscheider, Phys. Rev. Lett. 97, 176803 (2006).
${ }^{13}$ M. Strass, P. Hänggi, and S. Kohler, Phys. Rev. Lett. 95, 130601 (2005).

${ }^{14}$ O. Klein, Z. Phys. 53, 157 (1929).

${ }^{15}$ M. I. Katsnelson, K. S. Novoselov, and A. K. Geim, Nature Phys. 2, 620 (2006).

${ }^{16}$ J. Tworzydlo, B. Trauzettel, M. Titov, A. Rycerz, and C. W. J. Beenakker, Phys. Rev. Lett. 96, 246802 (2006).

${ }^{17}$ E. Prada, P. San-Jose, and H. Schomerus, Phys. Rev. B 80, 245414 (2009).

${ }^{18}$ S. Kohler, J. Lehmann, and P. Hänggi, Phys. Rep. 406, 379 (2005).

${ }^{19}$ M. Wagner and F. Sols, Phys. Rev. Lett. 83, 4377 (1999).

${ }^{20}$ M. Wagner, Phys. Rev. A 51, 798 (1995).

${ }^{21}$ B. Trauzettel, Y. M. Blanter, and A. F. Morpurgo, Phys. Rev. B 75, 035305 (2007); 83, 159902(E) (2011).

${ }^{22}$ M. A. Zeb, K. Sabeeh, and M. Tahir, Phys. Rev. B 78, 165420 (2008); 79, 089903(E) (2009).

${ }^{23}$ Y. Gu, Y. H. Yang, J. Wang, and K. S. Chan, J. Phys. Condens. Matter 21, 405301 (2009).

${ }^{24}$ L. E. F. Foa Torres and G. Cuniberti, C. R. Phys. 10, 297 (2009).

${ }^{25}$ L. E. F. Foa Torres, H. L. Calvo, C. G. Rocha, and G. Cuniberti, Appl. Phys. Lett. 99, 092102 (2011).

${ }^{26}$ G. Stefanucci, S. Kurth, A. Rubio, and E. K. U. Gross, Phys. Rev. B 77, 075339 (2008). 